J3eA, Journal sur l'enseignement des sciences et technologies de l'information et des systèmes, Volume 4, Hors-Série 2, 11 (2005)

DOI : http://dx.doi.org/10.1051/bib-j3ea:2005711

(C) EDP Sciences, 2005

\title{
Commande fréquentielle en cap d'un robot sous-marin
}

\author{
J.P. Folcher \\ UFR des Sciences \\ Laboratoire I3S \\ Université de Nice Sophia-Antipolis \\ 2000 route de Lucioles \\ BP 121 \\ F-06903 Sophia-Antipolis CEDEX, France
}




\title{
Commande fréquentielle en cap d'un robot sous-marin
}

\author{
Jean-Pierre Folcher \\ UFR des Sciences - Laboratoire I3S, Université de Nice - Sophia Antipolis \\ 2000, route des Lucioles, BP 121, F-06903 Sophia-Antipolis Cedex \\ folcher@i3s.unice.fr
}

Résumé : Cet article présente deux séances de travaux pratiques enseignées en master STIC de l'Université de Nice - Sophia Antipolis. L'objectif de ce projet est l'étude d'une méthode de synthèse $\mathbf{H}_{\infty}$ et la résolution d'un problème de commande d'un robot sous-marin.

Mots clés : commande robuste, commande $\mathbf{H}_{\infty}$, commande anti-windup, critère du cercle, robotique sous-marine.

\section{Introduction}

Au cours des années 80, la notion de robustesse (l'aptitude d'une loi de commande à garantir la stabilité, les performances sur un système réel) est devenue une préoccupation centrale. Les méthodes de synthèse basées sur l'optimisation d'un critère $\mathbf{H}_{\infty}$ ont permis de répondre à cette demande et de satisfaire un cahier des charges exprimé dans le domaine fréquentiel. L'utilisation de ces méthodes pour résoudre de nombreux problèmes industriels (contrôle d'attitude de satellites, pilotage de missiles) souligne leur intérêt et leur maturité. Il est important d'enseigner ces méthodes de commande en école d'ingénieur ou en master au même titre que les méthodes d'automatique classique et de commande optimale. Cet enseignement est déjà assuré dans plusieurs institutions d'enseignement, voir $[1,5,6]$.

Dans le cadre des enseignements de l'Université de Nice - Sophia Antipolis, un mini-projet illustrant cette méthode de synthèse est proposé aux étudiants. Des élèves-ingénieurs de l'Ecole des Mines de Paris ont également traité ce problème. Ce projet permet de concevoir le système de commande en cap d'un robot sous-marin. Les saturations en vitesse des propulseurs sont prises en compte explicitement par une méthode de commande comprenant deux étapes :

- synthèse d'un correcteur nominal assurant des performances en négligeant les non-linéarités,

- synthèse d'un correcteur anti-windup permettant d'assurer la stabilité absolue du système incluant une non-linéarité.

\section{Modélisation du véhicule}

Ce véhicule (voir Fig.1) est actionné par deux propulseurs horizontaux pour les degrés de liberté longitudinal et de cap et par un propulseur vertical pour le déplacement en profondeur. Les dynamiques en roulis et en assiette ne sont pas commandées mais sont naturellement stables.

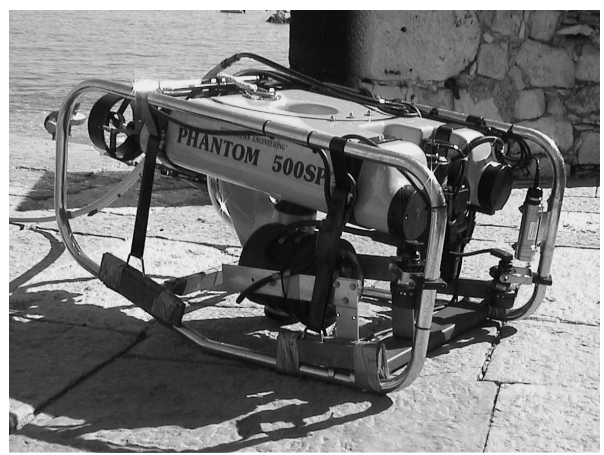

FIG. 1 - Le robot Phantom 500.

L'utilisation de modèles découplés pour décrire les mouvements du robot permettent de simplifier les étapes d'identification (à l'aide des capteurs à bord du robot) et de synthèse des correcteurs. Les dynamiques du mouvement horizontal du robot sont déduites des équations générales de Newton-Euler exprimées dans le repère mobile du robot, voir [3]. La dynamique du mouvement du robot avec un précompensateur est représentée sur la figure 2. Une modélisation plus détaillée du mouvement de ce véhicule est donnée en [2]. Les mouvements en cap (angle $\psi$ ) et en vitesse longitudinale $u$ sont engendrés par les signaux $a_{r}$ et $a_{u}$. Ces signaux, à un facteur multiplicatif près (inerties), représentent respectivement le couple et l'effort produits par les propulseurs gauche et droit. Ces derniers sont commandés en vitesse. La fonction liant les accélérations aux vitesses de rotation des propulseurs $n_{g}$ et $n_{d}$ est non linéaire. Un précompensateur (voir [2]) permet de découpler et de linéariser les deux dynamiques. Pour le régime stationnaire, les signaux $a_{r}$ et $a_{u}$ atteignent les valeurs des signaux de référence $a_{r}^{r}$ et $a_{u}^{r}$. Dans la suite du projet, on fixe une accélération longitudinale de référence nulle $a_{u}^{r}=0$. La saturation en vitesse des deux propulseurs induit un comportement non linéaire global de la dynamique horizontale pilotée par le précompensateur. 


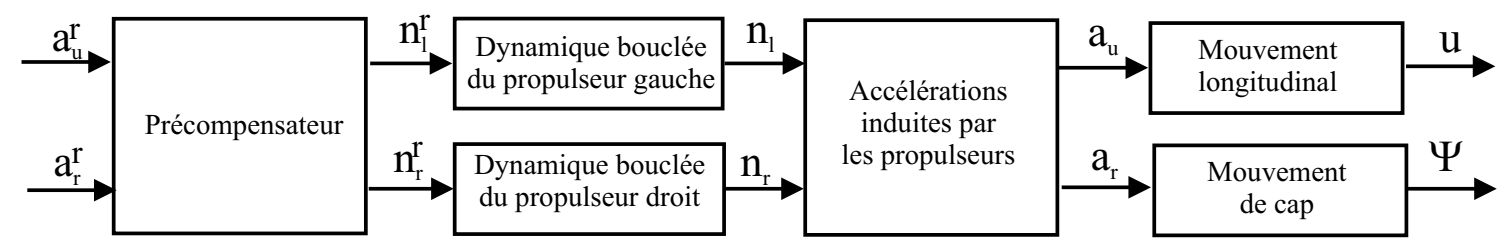

FIG. 2 - Dynamique horizontale du robot avec le précompensateur.

Le comportement de ce nouveau système peut être approximé (voir [2]) par les équations suivantes

$$
\begin{gathered}
v=\varphi(u) \\
\frac{\psi(p)}{v(p)}=G(p)=\frac{1}{p\left(\alpha_{r} p+1\right)} .
\end{gathered}
$$

La caractéristique de cette non-linéarité statique est représentée sur la figure 3.

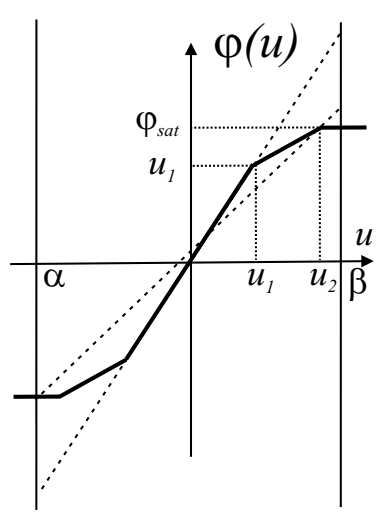

FIG. 3 - Non linéarité statique.
Les valeurs des paramètres $\alpha_{r}, u_{1}, u_{2}$ et $\varphi_{\text {sat }}$ sont précisées dans [2]. La fonction $\varphi$ satisfait à la condition de secteur $\left[\begin{array}{ll}a & b\end{array}\right]$ :

$(\varphi(t, q)-a q)(\varphi(t, q)-b q) \leq 0, \quad \forall t \geq 0, \forall q \in[\alpha \beta]$

Pour $|u(t)| \leq u_{\max }$, la fonction $\varphi$ vérifie la condition de secteur $\left[\frac{\bar{\varphi}_{\text {sat }}}{u_{\max }} 1\right]$.

\section{Conception du système de commande en cap}

\subsection{Structure de la loi de commande}

Le système de commande en cap du robot va comprendre le précompensateur non linéaire et une partie linéaire qui sera déterminée dans cette section. Sa structure est représentée sur la figure 4. $K_{n}(p)$ est le correcteur nominal assurant des performances lorsque la non-linéarité n'est pas « excitée » c.a.d. que $|u(t)| \leq u_{1}$, voir la figure 3 . Notons que dans ce cas le signal d'entrée du correcteur $K_{a w}(p)$ est nul ainsi que sa sortie : seul le correcteur $K_{n}(p)$ contribue à la réponse du système bouclé. Le correcteur $K_{a w}(p)$ agit quand la non-linéarité est « excitée »c.a.d. que $|u(t)|>u_{1}$. Il stabilise le système bouclé lorsque la fonction $\varphi$ vérifie les conditions de secteur (3).

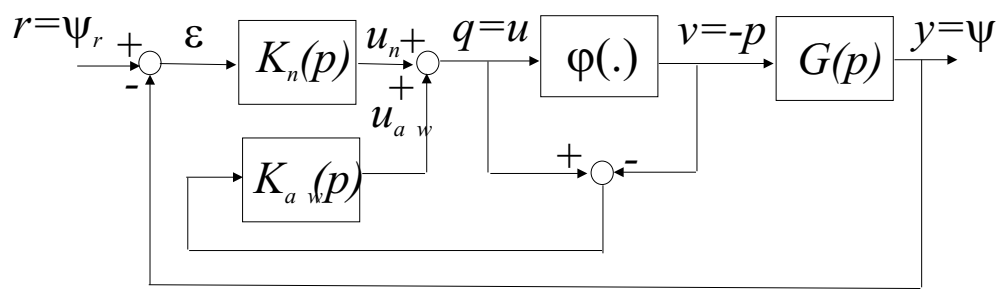

FIG. 4 - Structure de la loi de commande.

\subsection{Synthèse du correcteur nominal}

On considère que $|u(t)| \leq u_{1}$ et donc que $v=$ $\varphi(u)=u$. En enlevant le correcteur $K_{a w}(p)$, le système bouclé peut être mis sous la forme $\mathbf{H}_{\infty}$-standard présentée dans $[1,5]$. Soit $T_{z w}(p)$ la matrice de transfert entre les signaux $z$ et $w$ représentés sur la figure 5 . La méthode de Glover and Doyle basée sur la résolution de deux équations de Riccati permet de déterminer le correcteur $K_{n}$ qui stabilise le système bouclé et qui impose :

$$
\left\|T_{z w}(p)\right\|_{\infty}=\left\|\begin{array}{l}
W_{1}(p) T_{\epsilon r}(p) \\
W_{2}(p) T_{u r}(p)
\end{array}\right\|_{\infty}<1 .
$$

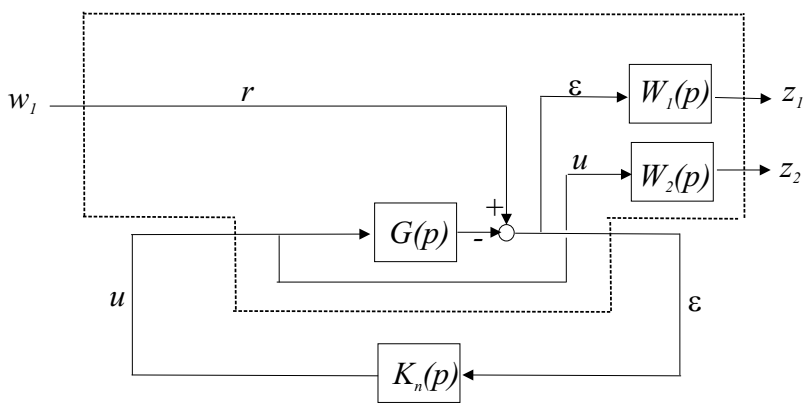

FIG. 5 - Problème de synthèse de $K_{n}$ sous la forme $\mathbf{H}_{\infty}$ standard. 
Par conséquent, les contraintes fréquentielles suivantes sont assurées pour toutes les pulsations $\omega$

$$
\begin{aligned}
& \left|T_{\epsilon r}(j \omega)\right|=\left|\frac{1}{1+G(j \omega) K_{n}(j \omega)}\right|<\gamma\left|W_{1}(j \omega)\right|^{-1}, \\
& \left|T_{u r}(j \omega)\right|=\left|\frac{K_{n}(j \omega)}{1+G(j \omega) K_{n}(j \omega)}\right|<\gamma\left|W_{2}(j \omega)\right|^{-1},
\end{aligned}
$$

et peuvent être vérifiées graphiquement sur les figures 6 et 7 .

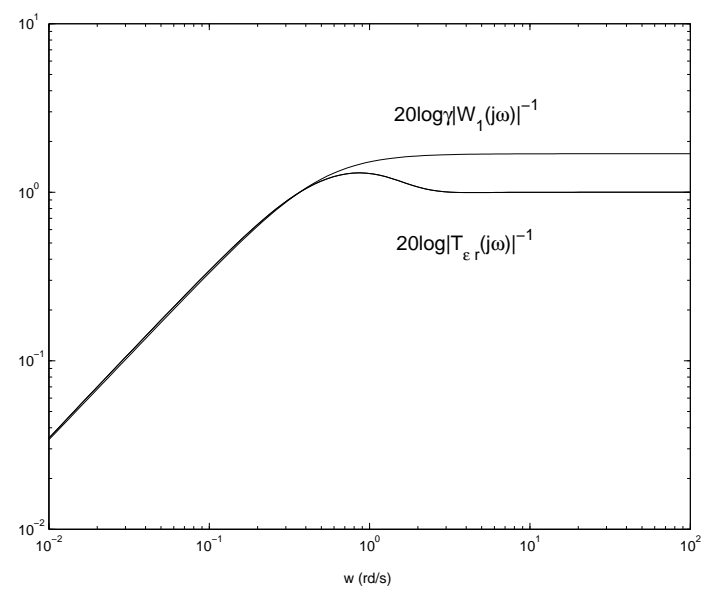

FIG. 6 - Diag. de Bode de $T_{\epsilon r}(p)$ et de $W_{1}(p)^{-1}$.

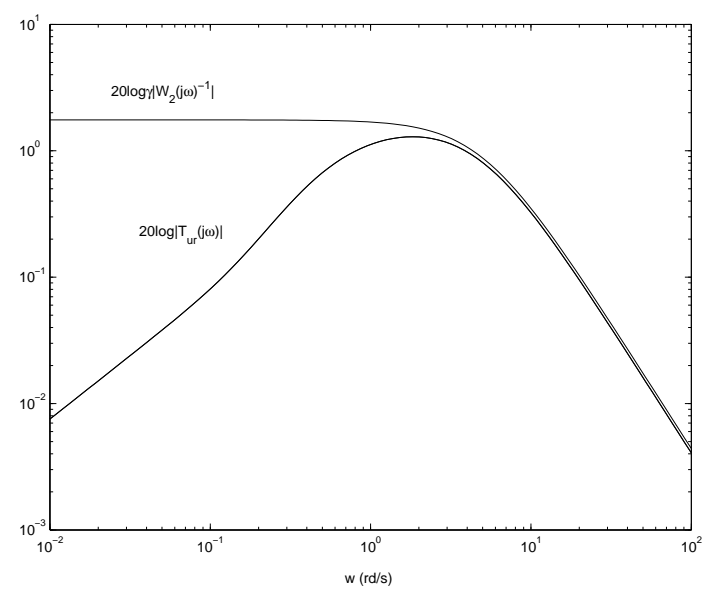

FIG. 7 - Diag. de Bode de $T_{u r}(p)$ and $W_{2}(p)^{-1}$.

\subsection{Synthèse du correcteur anti-windup}

L'objectif de la synthèse du correcteur $K_{a w}(p)$ est d'assurer la stabilité lorsque la saturation est « exitée » c.a.d que $|u(t)|>u_{1}$, voir la figure 3 . Le système bouclé représenté sur la figure 4 peut être réinterprété comme l'interconnection entre un système linéaire (défini par sa fonction de transfert $T_{q p}$ ) et la fonction non linéaire $\varphi$ qui vérifie la condition de secteur (3).

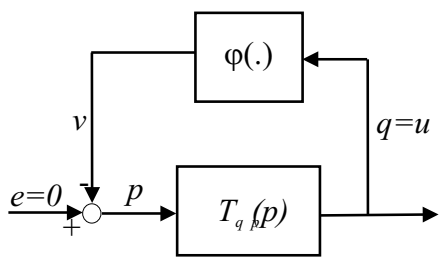

FIG. 8 - Système interconnecté.

Le critère du cercle (voir [4]) permet d'analyser la stabilité de ce système interconnecté $\left(T_{q p}, \varphi\right)$ dans le cas où $0<a<b$. La fonction de transfert $T_{q p}(p)$ doit être stable et son lieu de Nyquist ne doit pas rentrer dans le cercle $D(a, b)$, voir la figure 9 .

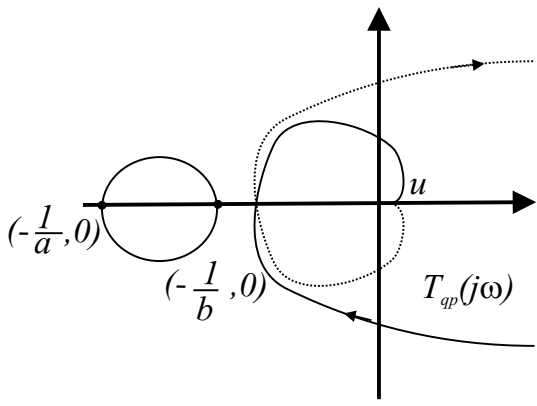

FIG. 9 - Lieu de Nyquist de $T_{q p}(p)$.

Une transformation de boucle permet de définir une nouvelle interconnection $\left(\bar{T}_{\bar{q} \bar{p}}, \bar{\varphi}\right)$ où la nouvelle non-linéarité

$$
\bar{\varphi}(\bar{q})=\left.\Pi^{-1}(\varphi(q)-\Gamma q)\right|_{q=\bar{q}}
$$

appartient au secteur $[-11]$, avec $\Gamma=\frac{b-a}{2}$ et $\Pi=\frac{a+b}{2}$. Pour que la stabilité soit assurée, le critère du cercle impose que la fonction de transfert

$$
\bar{T}_{\bar{q} \bar{p}}(p)=\left(1+T_{q p}(p) \Gamma\right)^{-1} T_{q p}(p) \Pi
$$

vérifie $\left\|\bar{T}_{\bar{q} \bar{p}}(p)\right\|_{\infty}<1$. Il est possible d'imposer cette contrainte en utilisant la forme standard représentée sur la figure 10.

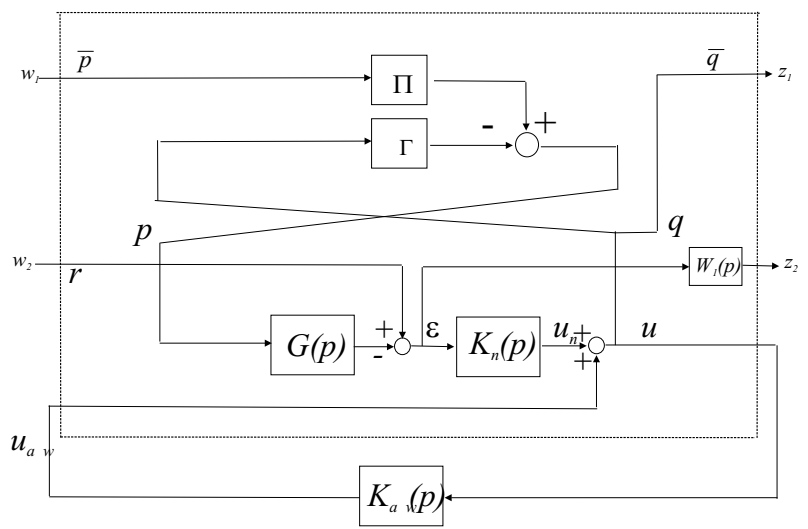

FIG. 10 - Problème de synthèse de $K_{a w}$ sous la forme $\mathbf{H}_{\infty}$ standard. 
On recherche donc le correcteur $K_{a w}(p)$ qui impose la condition :

$$
\left\|\bar{T}_{z w}(p)\right\|_{\infty}=\left\|\begin{array}{cc}
\bar{T}_{\bar{q} \bar{p}}(p) & T_{\bar{q} r}(p) \\
\bar{W}_{1}(p) \bar{T}_{\epsilon \bar{p}}(p) & \bar{W}_{1}(s) \bar{T}_{\epsilon r}(s)
\end{array}\right\|_{\infty}<1
$$

Par conséquent $\left\|\bar{T}_{\bar{q} \bar{p}}(p)\right\|_{\infty}<1$, la stabilité absolue est imposée. La contrainte fréquentielle

$$
\left\|\bar{W}_{1}(p) \bar{T}_{\epsilon r}(p)\right\|_{\infty}<\bar{\gamma},
$$

sur la fonction de sensibilité est satisfaite. On peut vérifier graphiquement ces contraintes.

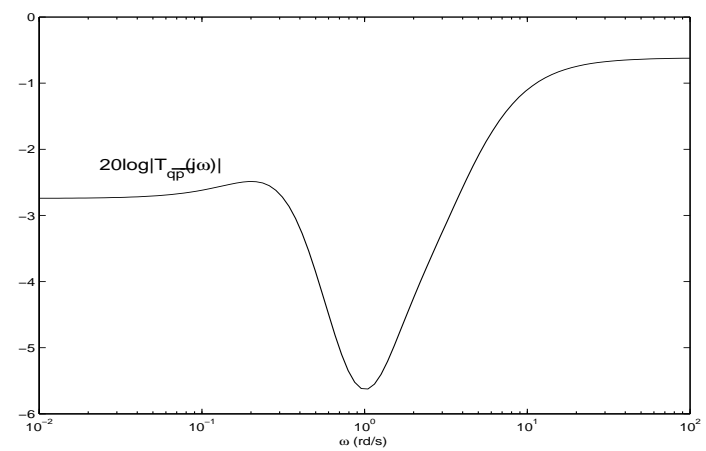

FIG. 11 - Diagramme de Bode de $\bar{T}_{\bar{q} \bar{p}}(p)$.

$\bar{T}_{\epsilon r}^{k}(p)$ est la fonction de transfert décrivant la relation entre les signaux $r$ et $\epsilon$ definis sur la figure 4 pour $\varphi(u)=k u$ avec $k \in\left[\begin{array}{ll}a & b\end{array}\right]$ un gain fixé. Remarquons que la contrainte fréquentielle de performance imposée sur $\bar{T}_{\epsilon r}^{k}(p)$ est assurée de manière robuste c.a.d pour tout $k \in[a b]$.

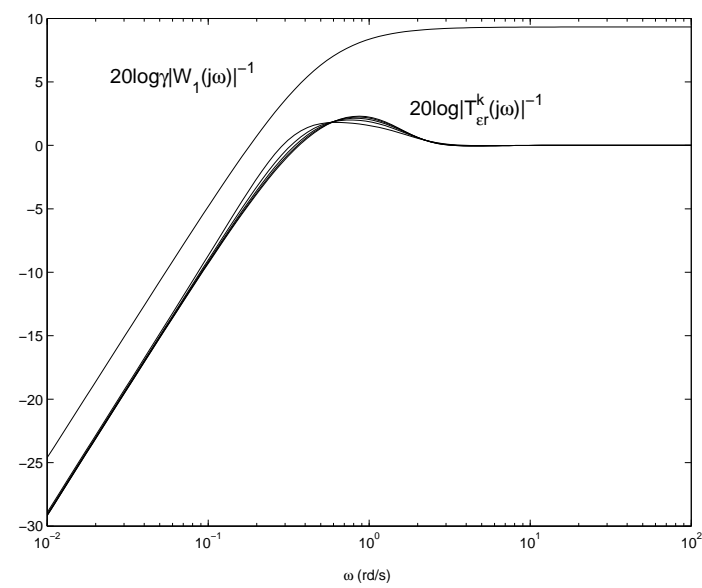

FIG. 12 - Diag. de Bode de $\bar{T}_{\epsilon r}^{k}(p)$ et de $\bar{W}_{1}(p)^{-1}$.

\subsection{Simulation temporelle}

La sortie $\psi$ du système lorsque l'entrée de référence est un échelon de hauteur $180^{\circ}$ est tracée sur la figure 13. Le temps de réponse est de $16 s$ et la réponse temporelle n'a pas de dépassement. L'évolution de l'entrée de commande $u$ est représentée sur la figure 14. Il est à noter que la commande $u_{a w}$ est nulle lorsque la non-linéarité n'est pas « exitée » i.e. quand $|u(t)|<u_{1}$.

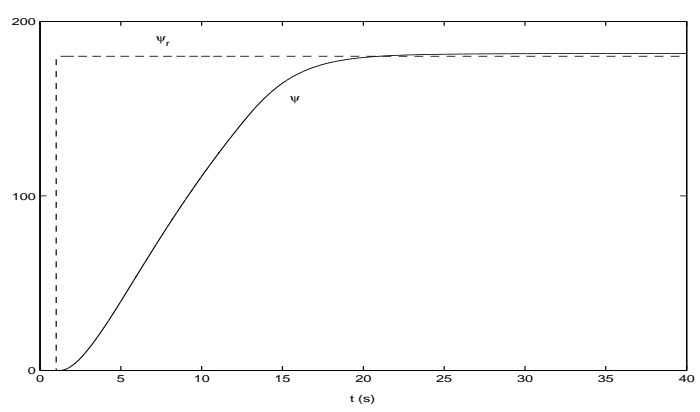

FIG. 13 - Réponse indicielle de la sortie $\psi$.

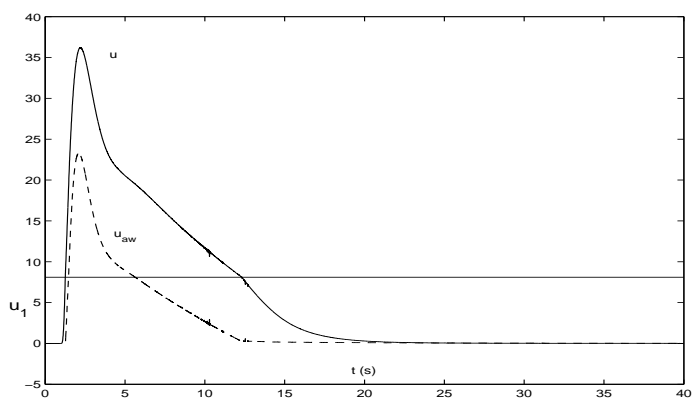

FIG. 14 - Réponse indicielle des signaux de commande $u$ et $u_{a w}$.

\section{Références}

[1] G. Duc et S. Font. Commande $\mathbf{H}_{\infty}$ et $\mu$ analyse : des outils pour la robustesse. Hermès, 1999.

[2] J.P. Folcher. Steering control of an underwater vehicle : an anti-windup design. 13th International Symposium on Unmanned Untethered Submersible Technology, august 2003.

[3] T.I. Fossen, Guidance and Control of Ocean Vehicles. John Wiley \& Sons, 1995.

[4] H. K. Khalil. Nonlinear Systems. Prentice Hall, second edition, 1996.

[5] G. Scorletti et V. Fromion. Introduction à la commande multivariable des systèmes : méthodes de synthèse fréquentielle $\mathbf{H}_{\infty}$, polycopié de cours de l'ENSI de Caen, septembre 2001.

[6] G. Scorletti, G. Duc, S. Font, V. Fromion et J.-P. Folcher. Automatique fréquentielle avancée : apprentissage d'un système mécanique flexible, Colloque CETSIS-EEA, Montpellier, pp.119-121, novembre 1999.

[7] J.P. Folcher et J. Rendas. Une plateforme expérimentale pour l'automatique : le robot sous-marin Phantom 500. Colloque CETSISEEA, Clermont-Ferrand, pp.149-152, novembre 2001. 